\title{
Few insights on the post-COVID-19 problem
}

\author{
Alberca GGF, Alberca RW \\ Laboratorio de Dermatologia e Imunodeficiencias (LIM-56), Departamento de Dermatologia, Faculdade de Medicina FMUSP, \\ Universidade de São Paulo, São Paulo, Brazil. ricardowesley@usp.br
}

KEY WORDS: COVID-19, SARS-CoV-2, long-term, sequelae, immune response.

\section{Dear Editor,}

We read with great interest the article entitled "Few Insights on the problem of COVID-19” recently published by El Hassoun et al. in the Bratislava Medical Journal. The manuscript highlights the clinical characteristics, disease mechanisms, treatment perspectives, and public and social issues of the coronavirus disease - 2019 (COVID-19) (1). Since COVID-19 has affected millions worldwide we would like to add a few points.

COVID-19 is a systemic disease caused by infection with severe acute respiratory syndrome coronavirus-2 (SARS-CoV-2). COVID-19 patients can experience a range of clinical symptoms, from no symptoms to critical illness, with respiratory discomfort and multi-organ failure.

In patients with severe illness, a systemic inflammatory response is triggered by the infection, evolving to a potentially fatal, organic dysfunction caused by an inadequate or dysregulated host response to infection and abnormal coagulation. Leading to the hypothesis that SARS-CoV-2 can cause a viral sepsis $(1,2)$.

A recent report identified that after COVID-19 patients can present persistent symptoms like dyspnea, fatigue, and joint pain (3). These alterations could be due to injuries caused by the immune response to COVID-19, especially in the respiratory system, but fail to explain joint pain.

Akter et al identified metabolic alteration in patients with Diabetes Mellitus, with an increase in the necessity for the use of insulin and an increase in dosage for patients already insulin-dependent (4). Therefore, COVID-19 may also harm blood glucose control in the general population.

Another report identified immune sequelae post-COVID-19, with alterations on the frequency of subpopulations two months

Laboratorio de Dermatologia e Imunodeficiencias (LIM-56), Departamento de Dermatologia, Faculdade de Medicina FMUSP, Universidade de São Paulo, São Paulo, Brazil

Address for correspondence: R. Wesley Alberca, Dr, Laboratorio de Dermatologia e Imunodeficiencias (LIM-56), Departamento de Dermatologia, Faculdade de Medicina FMUSP, Universidade de São Paulo, Av. Dr. Enéas Carvalho de Aguiar, 470, São Paulo, 05403-000 Brazil.

Phone: +55.11.999747495

Acknowledgement: Source(s) of support in the form of grants: RWA has a fellowship from Fundação de Amparo à Pesquisa do Estado de São Paulo (FAPESP) grant: 19/02679-7. after SARS-CoV-2 clearance (5). In the short or long-term these immunological alterations could represent an elevated risk for other infections.

Due to the gap in the current knowledge about the range and severity of the long-term effects of COVID-19, we believe that investigations on the long-lasting effects of COVID-19 and survival post-COVID-19 are necessary, and may represent a future health challenge worldwide.

\section{References}

1. El Hassoun O, Valaskova Z, Polak S, Hulin I. Few insights on the problem of COVID-19. Bratisl Med J Published Online First: 2020. DOI: 10.4149/BLL_2020_078.

2. Li H, Liu L, Zhang D, Xu J, Dai H, Tang N et al. SARS-CoV-2 and viral sepsis: observations and hypotheses. Lancet 2020. DOI: 10.1016/ S0140-6736(20)30920-X.

3. Carfi A, Bernabei R, Landi F. Persistent symptoms in patients after acute COVID-19. JAMA - J. Am Med Assoc 2020. DOI: 10.1001/ jama.2020.12603.

4. Akter F, Mannan A, Mehedi HMH, Rob MA, Ahmed S, Salauddin A et al. Clinical characteristics and short term outcomes after recovery from COVID-19 in patients with and without diabetes in Bangladesh. Diabetes Metab Syndr Clin Res Rev Published Online First: 2020. DOI: 10.1016/j.dsx.2020.10.016.

5. Orologas-Stavrou N, Politou M, Rousakis P, Kostopoulos I V., Ntanasis-Stathopoulos I, Jahaj E et al. Peripheral Blood Immune Profiling of Convalescent Plasma Donors Reveals Alterations in Specific Immune Subpopulations Even at 2 Months Post SARS-CoV-2 Infection. Viruses Published Online First: 2020. DOI: 10.3390/v13010026.

Received February 25, 2021. Accepted March 4, 2021. 